\title{
Magnetoelectric effect and magnetic phase diagram of a polar ferrimagnet $\mathrm{CaBaFe}_{4} \mathrm{O}_{7}$
}

\author{
V. Kocsis, ${ }^{1,2}$ Y. Tokunaga, ${ }^{2,3}$ S. Bordács, ${ }^{4,5}$ M. Kriener, ${ }^{2}$ A. Puri, ${ }^{6}$ U. Zeitler, ${ }^{6}$ Y. Taguchi, ${ }^{2}$ Y. Tokura, ${ }^{2,5,7}$ and I. Kézsmárki ${ }^{4}$ \\ ${ }^{1}$ MTA-BME Condensed Matter Research Group, Budafoki ut 8, 1111 Budapest, Hungary \\ ${ }^{2}$ RIKEN Center for Emergent Matter Science (CEMS), Wako, Saitama 351-0198, Japan \\ ${ }^{3}$ Department of Advanced Materials Science, University of Tokyo, Kashiwa 277-8561, Japan \\ ${ }^{4}$ Department of Physics, Budapest University of Technology and Economics and MTA-BME Lendület Magneto-optical \\ Spectroscopy Research Group, 1111 Budapest, Hungary \\ ${ }^{5}$ Quantum-Phase Electronics Center, Department of Applied Physics, University of Tokyo, Tokyo 113-8656, Japan \\ ${ }^{6}$ High Field Magnet Laboratory (HFML-EMFL), Radboud University Nijmegen, Toernooiveld 7, 6525 ED Nijmegen, The Netherlands \\ ${ }^{7}$ Department of Applied Physics, University of Tokyo, Hongo, Tokyo 113-8656, Japan
}

(Received 19 November 2015; published 29 January 2016)

\begin{abstract}
The magnetic phase diagram of a polar ferrimagnet $\mathrm{CaBaFe}_{4} \mathrm{O}_{7}$ with a magnetic easy axis has been investigated by measurements of magnetization, specific heat, and magnetoelectricity. A ferrimagnetic transition takes place at $T_{\mathrm{C} 1}=275 \mathrm{~K}$ within the orthorhombic phase followed by a second magnetic transition at $T_{\mathrm{C} 2}=211 \mathrm{~K}$. Below $T_{\mathrm{C} 2}$, successive metamagnetic transitions occur for magnetic fields applied perpendicular to the easy axis, implying a sequential emergence of magnetic states which are neither collinear nor coplanar. The observation of the static magnetoelectric effect was limited to temperatures below $120 \mathrm{~K}$ due to the conducting nature of the crystals at higher temperatures. The magnitude of the ferroelectric polarization shows large changes between the different field-induced magnetic phases. The low-field state is characterized by a large linear magnetoelectric coefficient of $\alpha_{c c}=39 \mathrm{ps} / \mathrm{m}$, while a gigantic polarization change of $\Delta P=850 \mu \mathrm{C} / \mathrm{m}^{2}$ is observed for $\mu_{o} H=14 \mathrm{~T}$ applied along the easy axis.
\end{abstract}

DOI: 10.1103/PhysRevB.93.014444

\section{INTRODUCTION}

The magnetoelectric (ME) effect, which is a consequence of the cross-coupling between spin and charge degrees of freedom, is one of the most desired properties of materials for modern applications. In a magnetoelectric material, the magnetization can be changed by the application of an electric field and the electric polarization can be controlled by a magnetic field [1-7]. Such materials are promising for the development of low-power-consumption spintronics, nonvolatile ME data storage [8,9], optical diodes [10,11], and high-sensitivity ac magnetic field sensors [12]. Recently intensive efforts have been focused on the fundamental understanding of multiferroicity in well-known material classes, such as the perovskiterelated oxides [2-4,6,7], akermanites [10,13-15], hexaferrites [16], and the polar skyrmion host lacunar spinels [17,18]. However, $\mathrm{BiFeO}_{3}$ is still the only well-characterized roomtemperature multiferroic compound [19-21], which triggers a chase for new materials possibly exhibiting multiferroicity near room temperature. Swedenborgites with the formula of $A \mathrm{BM}_{4} \mathrm{O}_{7}$ ( $M$ being the magnetic ion) represent a new class of polar magnets where the long-range ferrimagnetic order occurs at relatively high temperatures [22,23].

The investigation of magnetoelectric effects in $\mathrm{CaBaFe}_{4} \mathrm{O}_{7}$, the target material of the present study, is partly motivated by the strong magnetoelectric coupling reported for $\mathrm{CaBaCo}_{4} \mathrm{O}_{7}[24,25]$. A giant polarization change of $\Delta P=$ $17000 \mu \mathrm{C} / \mathrm{m}^{2}$ was observed along the $c$ axis in $\mathrm{CaBaCo}_{4} \mathrm{O}_{7}$ across the transition from the pyroelectric paramagnetic state to the pyroelectric ferrimagnetic phase at $T_{\mathrm{C}}=64 \mathrm{~K}$ [25]. $A b$ initio calculations combined with Landau theory showed that the electric polarization change can be attributed to exchange-striction effects [26]. A similar polarization change of magnetoelastic origin is expected in the case of $\mathrm{CaBaFe}_{4} \mathrm{O}_{7}$.
Moreover, in $\mathrm{CaBaFe}_{4} \mathrm{O}_{7}$ the magnetic ordering takes place near room temperature [23], $T_{\mathrm{C} 1}=275 \mathrm{~K}$, which is a good starting point to further enhance the temperature of the magnetic phase transition by fine-tuning the material composition.

Other interesting aspects of this material are its polar structure and the coexistence of different geometrically frustrated sublattices occupied with mixed-valence iron ions. As explained in detail below, the structure of $A B M_{4} \mathrm{O}_{7}$ swedenborgites is polar along the $c$ axis, and consists of a stacking of alternating triangular and kagome layers of the magnetic $M$ ions. The nominal valence state of the transition metal $(M)$ is $2.5+$, which may imply that $M^{2+}$ and $M^{3+}$ ions in a $1: 1$ ratio are distributed among the triangular and kagome layers, respectively. Shorter oxygen-oxygen bonds were reported $[23,27,28]$ around the metal ions in the triangular layers, indicating that one of the two extra holes is localized on the triangular-lattice plane, and the other hole is distributed over the three inequivalent sites in the kagome layer. Furthermore, the presence of charge order was suggested based on the rapid increase of electrical resistivity towards lower temperatures [29-31]. Although the way of charge disproportionation and the onset of charge order are still open issues in $\mathrm{CaBaFe}_{4} \mathrm{O}_{7}$ and $\mathrm{CaBaCo}_{4} \mathrm{O}_{7}$, they may have a strong influence on the dielectric properties of the system.

Due to the mixed valency of magnetic ions in swedenborgites, charge ordering, if it occurs, can strongly influence the spin order. $\mathrm{CaBaFe}_{4} \mathrm{O}_{7}$ and $\mathrm{CaBaCo}_{4} \mathrm{O}_{7}$ are the only members of this material family where long-range threedimensional magnetic order has been reported [22,23,28]. In $\mathrm{CaBaCo}_{4} \mathrm{O}_{7}$, first-principles calculations suggested that the strong orthorhombic distortion of the structure may partially release the geometrical frustration, leading to a ferrimagnetic order [32,33]. The orthorhombic distortion in $\mathrm{CaBaCo}_{4} \mathrm{O}_{7}$ may be induced by the Jahn-Teller active $\mathrm{Co}^{3+}$ ions with $e^{3} t_{2}^{3}$ 
(a)

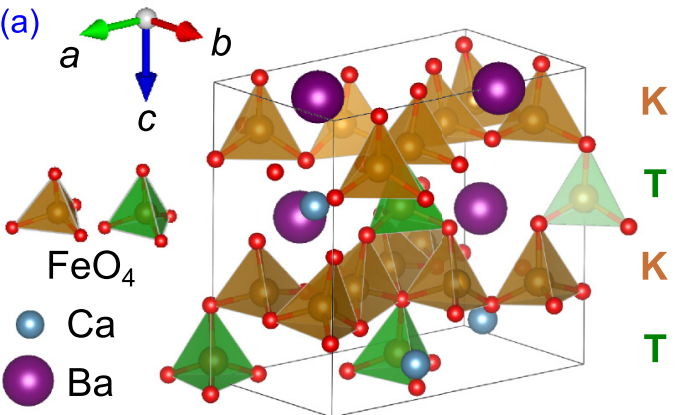

(b)

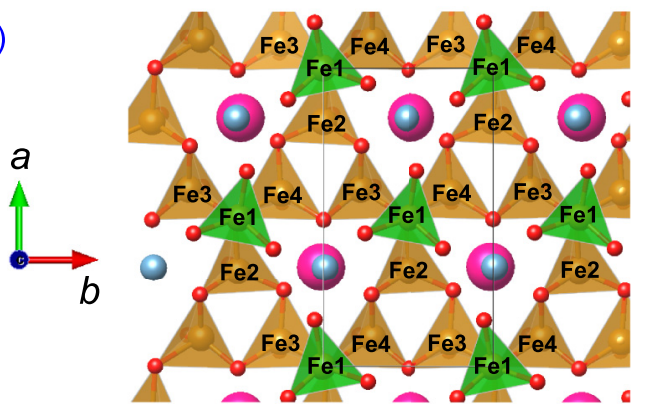

FIG. 1. (a) Orthorhombic unit cell of $\mathrm{CaBaFe}_{4} \mathrm{O}_{7}$ composed of 4 formula units. $\mathrm{FeO}_{4}$ tetrahedra belonging to the alternating kagome and triangular layers are indicated by brown and green colors, respectively $[23,43]$. The divalent $\mathrm{Ca}$ and $\mathrm{Ba}$ cations are located on top of each other in the voids of the stacking layers. (b) One kagome and one triangular layer as viewed from the crystallographic $c$ direction. The asymmetric shape of the tetrahedra, their misorientation relative to the main crystallographic directions of the regular triangular and kagome lattices, and the relative displacement of the $\mathrm{Ca}$ and $\mathrm{Ba}$ ions in the $a b$ plane are manifestations of the orthorhombic distortion.

configurations. This scenario is supported by the different temperature evolution of $\mathrm{Co}-\mathrm{O}$ bond lengths at different $\mathrm{Co}$ sites [32]. In its sister compounds with hexagonal symmetrysuch as $\mathrm{YbBaCo}_{4} \mathrm{O}_{7}, \mathrm{Ca}_{0.5} \mathrm{Y}_{0.5} \mathrm{BaCo}_{4} \mathrm{O}_{7}$, and $\mathrm{YBaFe}_{4} \mathrm{O}_{7}$ - the strong geometrical frustration fully suppresses the long-range magnetic order, and the compounds exhibit spin-glass or spin-liquid like ground states [28,34-39]. The complex and subtle nature of the magnetic state in these materials is well exemplified by the case of $\mathrm{YBaCo}_{4} \mathrm{O}_{7}$, which shows no long-range magnetic order, though its orthorhombic distortion should reduce the geometrical frustration of magnetic interactions [40-42].

The orthorhombic unit cell of $\mathrm{CaBaFe}_{4} \mathrm{O}_{7}$ with space group $P b n 2_{1}$ [23], shown in Fig. 1(a), is composed of four formula units. Although $\mathrm{CaBaFe}_{4} \mathrm{O}_{7}$ is orthorhombic at room temperature, we describe the main characteristics of its structure in comparison with its hypothetic high-symmetry form, the polar hexagonal $P 6_{3} m c$ structure, which is often realized in its sister compounds with solid solution of different transition metal ions at the $M$ sites [38,39,44,45]. The structure of $A B M_{4} \mathrm{O}_{7}$ consists of a stacking of alternating kagometriangular-kagome-triangular (KTKT) layers of magnetic $M$ ions with tetrahedral oxygen coordination. The corner-sharing oxygen tetrahedra are oriented with one vertex along the stacking direction of the KTKT layers, while the remaining three vertices lie in the $a b$ plane and are linked to those of neighboring tetrahedra, thereby giving rise to the polar structure. Adjacent kagome layers within KTKT units are rotated by $180^{\circ}$ around the $c$ axis with respect to each other, forming voids which are filled with the $A$ and $B$ cations. The stability of this structure is related not only to the size of the void and the ionic radii of the $A$ and $B$ cations, but also to the valence state of the $M$ ions $[39,42,46]$. For example, in the case of $\mathrm{CaBaCo}_{4} \mathrm{O}_{7}$, the presence of the Jahn-Teller active $\mathrm{Co}^{3+}$ ions leads to a structural phase transition to the $P b n 2_{1}$ orthorhombic symmetry by buckling and distortion of the $\mathrm{CoO}_{4}$ tetrahedra. In $\mathrm{CaBaFe}_{4} \mathrm{O}_{7}$ the Jahn-Teller distortion can emerge for the $\mathrm{Fe}^{2+}$ ions. The same structure was reported earlier for $\mathrm{CaBaFe}_{4} \mathrm{O}_{7}$ [23], where buckling of the $\mathrm{Fe}-\mathrm{O}_{4}$ tetrahedra leads to a rotation of the $\mathrm{Fe} 1-\mathrm{O}_{4}$ (green) tetrahedra and the asymmetry of the triangles formed by $\mathrm{Fe} 2-\mathrm{O}_{4}, \mathrm{Fe} 3-\mathrm{O}_{4}$, and $\mathrm{Fe} 4-\mathrm{O}_{4}$ tetrahedra, as shown in Fig. 1(b).

The primary purpose of this work is to investigate the complex magnetic structure of $\mathrm{CaBaFe}_{4} \mathrm{O}_{7}$ by revealing the magnetic phase diagram with the use of melt-grown crystals. As a further step, we present evidence for the large static magnetoelectric effect, which may arise from the interplay between the noncollinear and noncoplanar spin orders, the magnetostriction, and the mixed valency of the iron ions.

\section{EXPERIMENTAL TECHNIQUES}

\section{A. Sample preparation and characterization}

High-quality $\mathrm{CaBaFe}_{4} \mathrm{O}_{7}$ crystals were prepared by a conventional floating-zone technique. As the first step of the synthesis, a polycrystalline form of $\mathrm{CaBaFe}_{4} \mathrm{O}_{7}$ was prepared by a series of solid-state reactions. The main difficulty in the preparation of $\mathrm{CaBaFe}_{4} \mathrm{O}_{7}$ arises from the mixed-valence nature $\left(\mathrm{Fe}^{2+}\right.$ and $\left.\mathrm{Fe}^{3+}\right)$ of the target compound. In the sintering process, the average $2.5+$ valence state of the $\mathrm{Fe}$ ions was ensured by using the proper $2: 1$ stoichiometric ratio of $\mathrm{FeO}$ and $\mathrm{Fe}_{2} \mathrm{O}_{3}$ of the starting materials. Stoichiometric amounts of $\mathrm{CaCO}_{3}, \mathrm{BaCO}_{3}, \mathrm{FeO}$, and $\mathrm{Fe}_{2} \mathrm{O}_{3}$ were mixed and calcined in high-purity argon atmosphere for $12 \mathrm{~h}$ at $1000^{\circ} \mathrm{C}$, and after homogenization, the powder was further treated at $1100^{\circ} \mathrm{C}$ in high-vacuum atmosphere for $24 \mathrm{~h}$. The resultant material was pressed into a rod, which was further sintered at $1000^{\circ} \mathrm{C}$ in a reducing $\mathrm{Ar}+3 \% \mathrm{H}_{2}$ atmosphere for $12 \mathrm{~h}$. Single crystals were grown in flowing $\mathrm{N}_{2}$ atmosphere by an optical-imaging floating-zone apparatus.

The material was verified to be of a single phase by powder $\mathrm{x}$-ray diffraction and the crystal orientation was determined by Laue-diffraction patterns. While the $c$ axis is unique in our crystals, polarized optical microscopy showed the presence of structural twins with different orientations of the $a$ and $b$ axis. Magnetization and dielectric polarization measurements confirmed this observation, as no apparent anisotropy was detected in the $a b$ plane. Therefore, in the macroscopic quantities the structural twinning effectively masks the orthorhombicity and apparently restores the hexagonal symmetry of the crystals.

\section{B. Measurement techniques}

Magnetization measurements up to $\mu_{o} H=14 \mathrm{~T}$ and $32 \mathrm{~T}$ were respectively carried out in a physical property measurement system (Quantum Design) and at the High Field Magnetic 


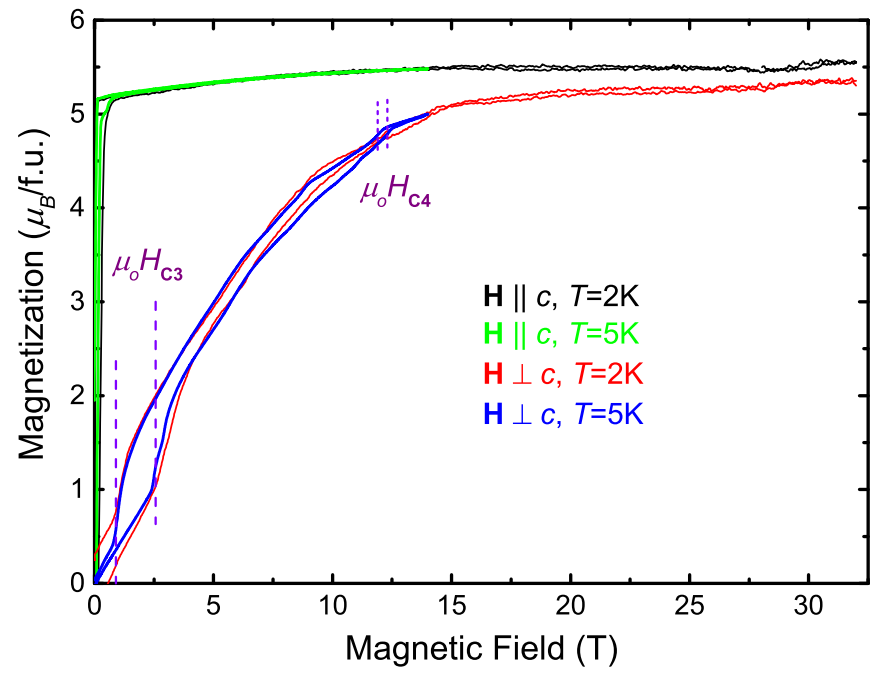

FIG. 2. Magnetic-field dependence of the magnetization measured in the low-temperature phase of $\mathrm{CaBaFe}_{4} \mathrm{O}_{7}$ for magnetic fields parallel (black and green) and perpendicular (red and blue) to the $c$ direction up to $\mu_{o} H=32 \mathrm{~T}$. The borders of the metamagnetic transitions are labeled by the $\mu_{o} H_{\mathrm{C} 3}$ lower critical and the $\mu_{o} H_{\mathrm{C} 4}$ upper critical fields, showing up for $\mathbf{H} \perp c$. Both are characterized by finite hysteresis.

Laboratory (HFML) in Nijmegen, respectively, using the vibrating sample magnetometer method in both cases. Specific heat was measured by a physical property measurement system (Quantum Design), applying a relaxation method in the temperature range of $T=2-300 \mathrm{~K}$, while for $T=150-600 \mathrm{~K}$ a Netzsch DSC 200 F3 differential scanning calorimeter equipped with a TASC 414/4 temperature controller was used.

Electric polarization measurements were performed on crystals with typical dimensions of $0.5 \times 4 \times 4 \mathrm{~mm}^{3}$ with a Keithley 6517A electrometer. Effective electric poling was practically prohibited, due to the high conductivity of the crystals even at $T_{\mathrm{C} 2}$. As a consequence, it was not possible to investigate the influence of magnetic ordering on the electric properties by means of pyrocurrent measurements. Instead, we carried out magnetocurrent measurements at fixed temperatures below $T=120 \mathrm{~K}$. (Above this temperature, leakage currents prevented the accurate measurement of fieldinduced polarization.) The magnetically induced polarization was determined by the integration of the magnetocurrent curves.

\section{RESULTS AND DISCUSSION}

\section{A. Magnetic properties}

Figure 2 shows the field dependence of the magnetization at low temperatures. In contrast to the easy-plane ferrimagnetic character of $\mathrm{CaBaCo}_{4} \mathrm{O}_{7}$ [22,32], $\mathrm{CaBaFe}_{4} \mathrm{O}_{7}$ is an easy-axis ferrimagnet with a spontaneous magnetization pointing along the $c$ axis [23]. The remanent magnetization is $M_{c}^{\mathrm{Rem}} \approx$ $2.5 \mu_{B}$ /f.u., which is remarkably large compared to other compounds in the swedenborgite family. For magnetic fields applied along the easy axis, accompanying a narrow hysteresis the magnetization reaches the value of $\sim 5.1 \mu_{B} /$ f.u. In the field range of $\mu_{o} H=14-28 \mathrm{~T}$, there is a broad magnetization

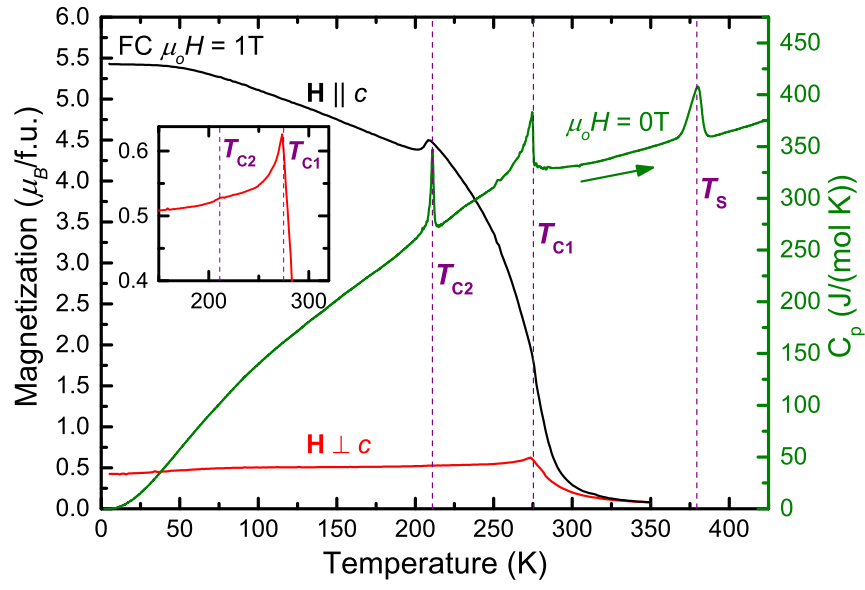

FIG. 3. Temperature dependence of the magnetization in field cooling (FC) with $\mu_{o} H=1 \mathrm{~T}$ applied along and perpendicular to the $c$ direction, and the specific heat of $\mathrm{CaBaFe}_{4} \mathrm{O}_{7}$ in zero field in a warming run. Three phase transitions are observed in the specific heat among which the one at $T_{\mathrm{S}}=380 \mathrm{~K}$ is likely to be of structural origin. The other two transitions are strongly manifested in the magnetization as well. $T_{\mathrm{C} 1}=275 \mathrm{~K}$ is the Curie temperature of the ferrimagnetic ordering, and $T_{\mathrm{C} 2}=211 \mathrm{~K}$ is a second magnetic transition, where magnetization along the $c$ axis decreases slightly. The inset shows a magnified view of the magnetization in the field-cooling run around $T_{\mathrm{C} 1}$ and $T_{\mathrm{C} 2}$ with $\mathbf{H} \perp c$.

plateau with a slightly larger moment of $M_{c}^{\text {Sat }} \approx 5.5 \mu_{B} /$ f.u. This value corresponds to about $1 / 3$ of the magnetization in the fully polarized state (18 $\mu_{B} /$ f.u.).

For magnetic fields applied perpendicular to the $c$ axis, successive metamagnetic transitions are observed between $\mu_{o} H=1$ and $14.5 \mathrm{~T}$ at $T=2 \mathrm{~K}$ [see also Fig. 5(d) and Fig. 6(e)]. Due to their broad hysteresis the subsequent metamagnetic transitions overlap with each other and it is not

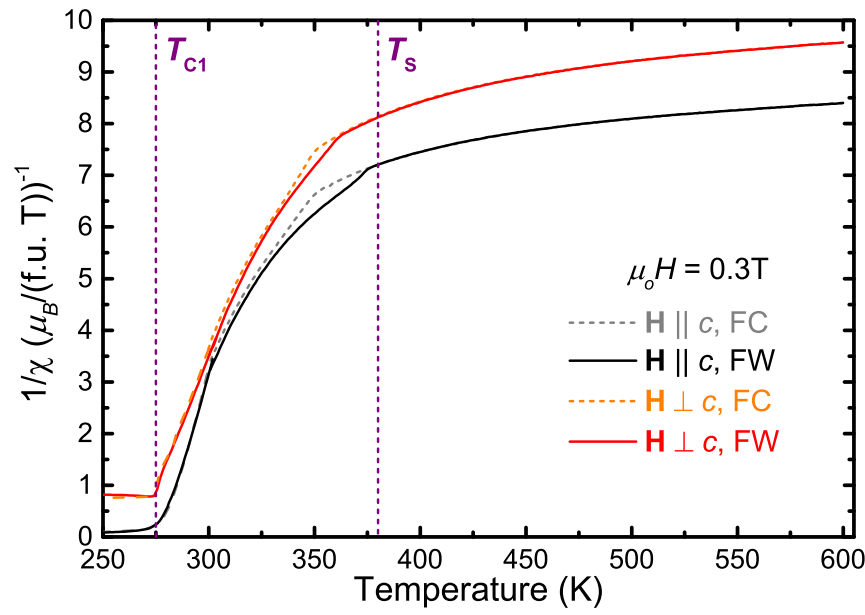

FIG. 4. Curie plot of the high-temperature magnetic susceptibility of $\mathrm{CaBaFe}_{4} \mathrm{O}_{7}$ with magnetic fields applied along and perpendicular to the $c$ axis, in field-cooling (FC) and field-warming (FW) runs. Vertical dashed lines indicate the structural transition temperature $\left(T_{\mathrm{S}}\right)$ as determined from the zero-field specific-heat measurements and the ferrimagnetic transition temperature $\left(T_{\mathrm{C} 1}\right)$. 
possible to unambiguously determine their critical field values, though their overlaps are reduced towards higher temperatures as can be followed in Fig. 6(e). Hereafter, we will refer to this field range as the metamagnetic region bound by a lower critical field $H_{\mathrm{C} 3}$ and an upper critical field $H_{\mathrm{C} 4}$. Above this field range the magnetization nearly approaches the value found for fields parallel to the easy axis, which indicates that the Zeeman energy overcomes the effect of easy-axis anisotropy for $\mu_{o} H \gtrsim 15 \mathrm{~T}$.

Figure 3 shows the temperature dependence of the magnetization measured in $\mu_{o} H=1 \mathrm{~T}$ and the zero-field specific heat. Three transitions are manifested in the specific-heat measurements at $T_{\mathrm{S}}=380 \mathrm{~K}, T_{\mathrm{C} 1}=275 \mathrm{~K}$, and $T_{\mathrm{C} 2}=211 \mathrm{~K}$. The anomaly at $T_{\mathrm{S}}=380 \mathrm{~K}$ likely indicates a structural transition as the material is still paramagnetic at this temperature. The other two transitions at $T_{\mathrm{C} 1}=275 \mathrm{~K}$ and $T_{\mathrm{C} 2}=211 \mathrm{~K}$, formerly described as a ferrimagnetic and a spin reorientation transition $[23,47,48]$, show up in the magnetization curves. At $T_{\mathrm{C} 2}$ there is only a weak anomaly in the magnetization measured in $\mu_{o} H=1 \mathrm{~T}$ applied within the $a b$ plane.
Figure 4 shows the inverse magnetic susceptibilities around and above room temperature, which were measured in $\mu_{o} H=$ $0.3 \mathrm{~T}$ magnetic field applied along and perpendicular to the $c$ axis. The uniaxial magnetic anisotropy with respect to the $c$ axis persists up to temperatures well above $T_{\mathrm{S}}=380 \mathrm{~K}$. For both orientations of the magnetic field the susceptibility curves exhibit anomalies near $T_{\mathrm{S}}=380 \mathrm{~K}$, the temperature of the structural transition as determined from the zero-field specific-heat measurement, and a broad hysteresis below this temperature. By fitting the $c$ axis and $a b$ plane susceptibilities with the Curie-Weiss law in the limited temperature range of $T=500-600 \mathrm{~K}$ one obtains $\theta_{c}=-2150 \mathrm{~K}$ and $\theta_{a b}=$ $-2050 \mathrm{~K}$, respectively, which point to a strong antiferromagnetic coupling among the $\mathrm{Fe}^{2+}$ and $\mathrm{Fe}^{3+}$ magnetic moments. The relatively large value of $\theta / T_{\mathrm{C} 1} \approx 7.7$ indicates the frustrated nature of the magnetic interactions.

The effective magnetic moments obtained for field parallel and perpendicular to the $c$ axis, $\mu_{\text {eff, } c}=22 \mu_{B} /$ f.u. and $\mu_{\text {eff }, a b}=20.4 \mu_{B} /$ f.u., are nearly comparable with the value $2\left(2 \sqrt{2 \times 3}+2 \sqrt{\frac{5}{2} \times \frac{7}{2}}\right) \mu_{B} /$ f.u. $=21.6 \mu_{B} /$ f.u. expected for
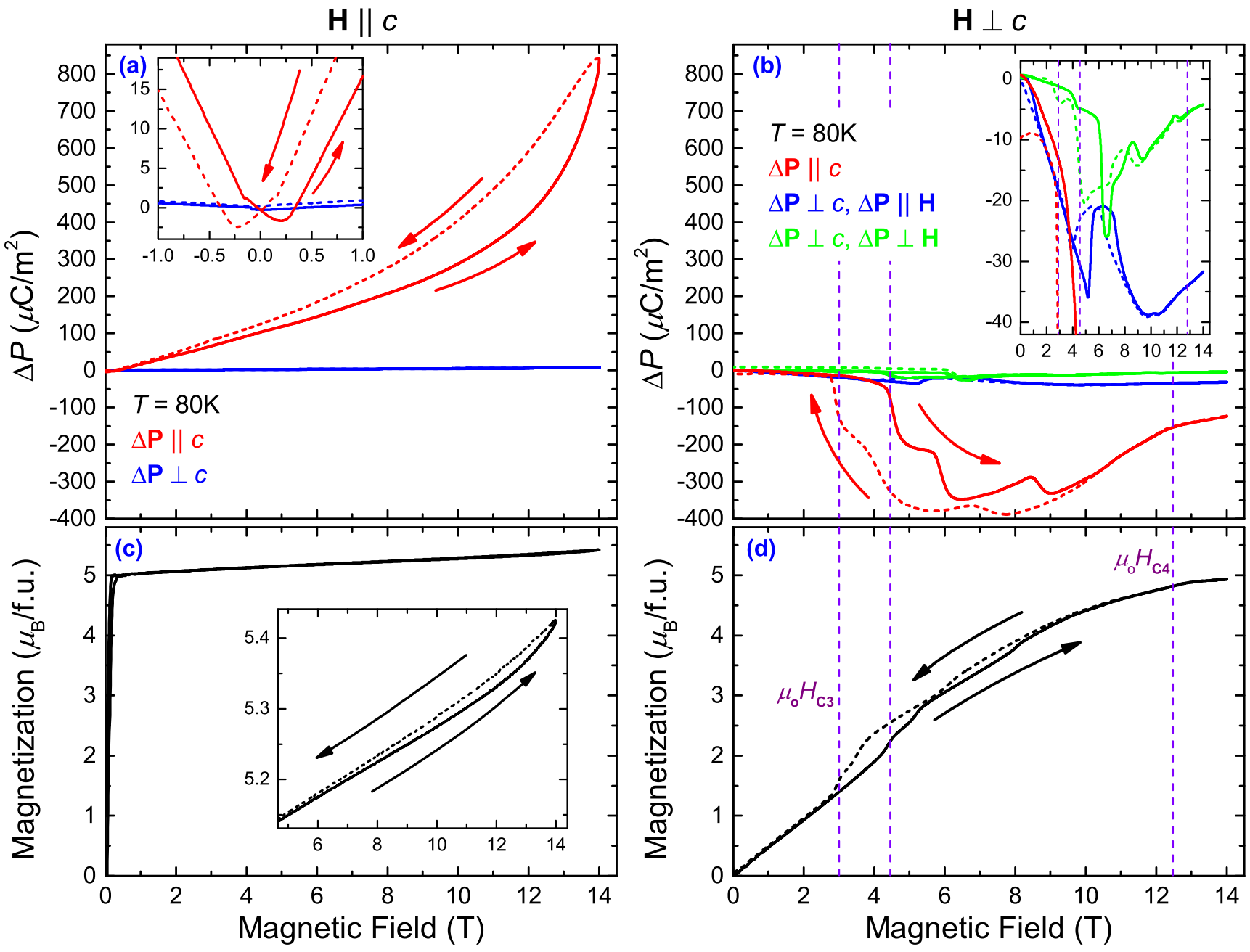

FIG. 5. Magnetic-field dependence of the polarization change and corresponding magnetization curves for magnetic fields applied along the $c$ axis [panels (a) and (c)] and within the $a b$ plane [panels (b) and (d)] at $T=80 \mathrm{~K}$. The largest change was observed in the $c$ component of the polarization irrespective of the orientation of the magnetic field. The inset to (a) shows the symmetric butterfly loop in $\Delta \mathbf{P} \| c$ in the $-1 \mathrm{~T} \leqslant \mu_{o} H \leqslant 1 \mathrm{~T}$ region. The inset to (b) shows the polarization changes perpendicular to the $c$ axis plotted in an enlarged scale. In the inset of (c), $c$-axis magnetization is magnified to show the hysteresis corresponding to that of the polarization change. 
the four mixed-valence Fe ions per formula unit. The frustrated antiferromagnetic interactions, together with the saturation moment of $M_{c}^{\text {Sat }} \approx 5.5 \mu_{B} /$ f.u., is indicative of a noncollinear or noncoplanar magnetic structure, as already proposed in a former X-ray magnetic circular dichroism study [23].

\section{B. Magnetoelectric properties}

Figure 5 summarizes the magnetic-field dependence of different components of the ferroelectric polarization at
$T=80 \mathrm{~K}$ for different orientations of the magnetic field. For a direct comparison between the magnetic and magnetoelectric properties of $\mathrm{CaBaFe}_{4} \mathrm{O}_{7}$, the corresponding magnetization data are simultaneously plotted in the lower panels of the figure.

Figure 5(a) shows the field-induced polarization $\Delta P$ when the magnetic field is applied along the $c$ axis. The polarization component parallel to the field is as large as $\Delta P=$ $850 \mu \mathrm{C} / \mathrm{m}^{2}$ in $\mu_{o} H=14 \mathrm{~T}$, while the polarization component (a)
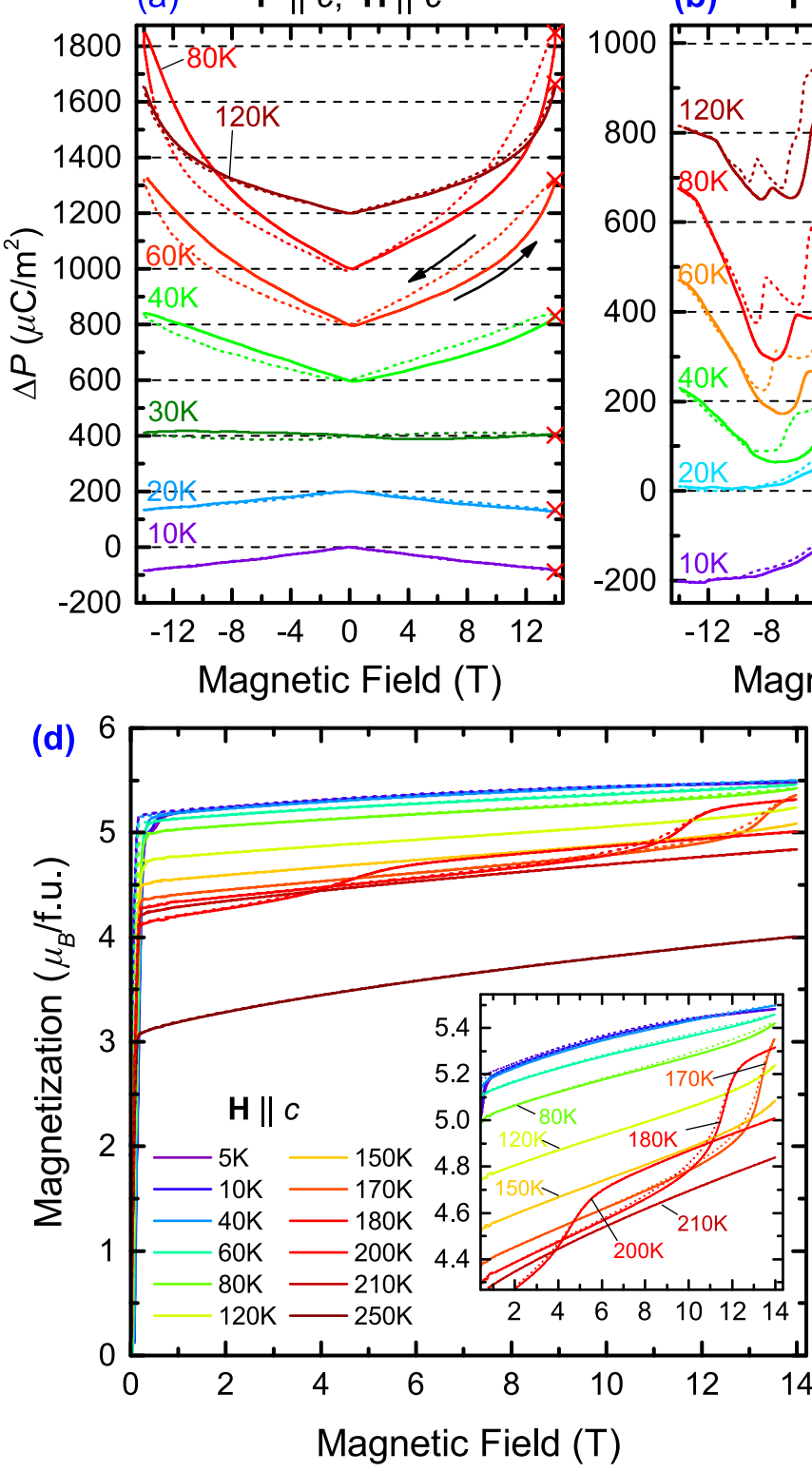

(b) $\quad \mathbf{P} \| c, \mathbf{H} \perp c$

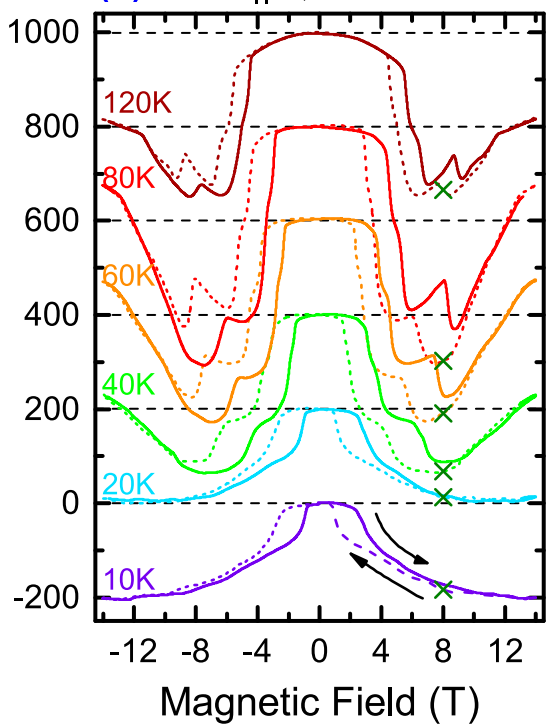

(c) $\mathbf{P} \| \mathbf{H}, \mathbf{H} \perp \mathbf{C}$

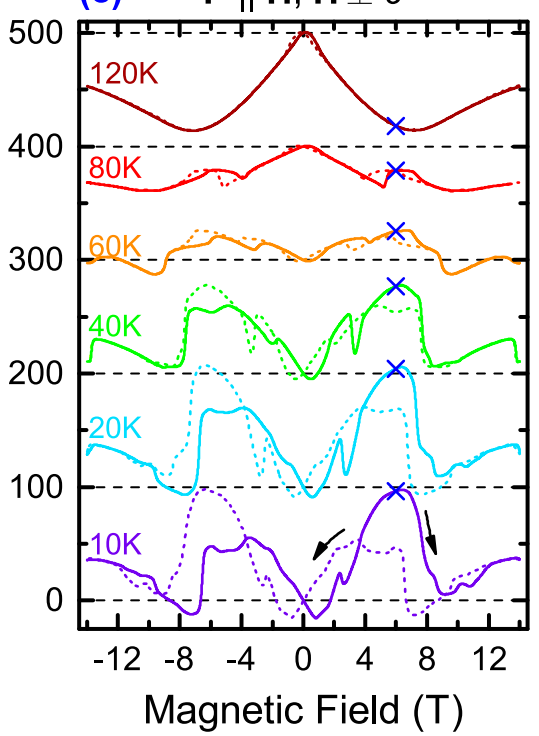

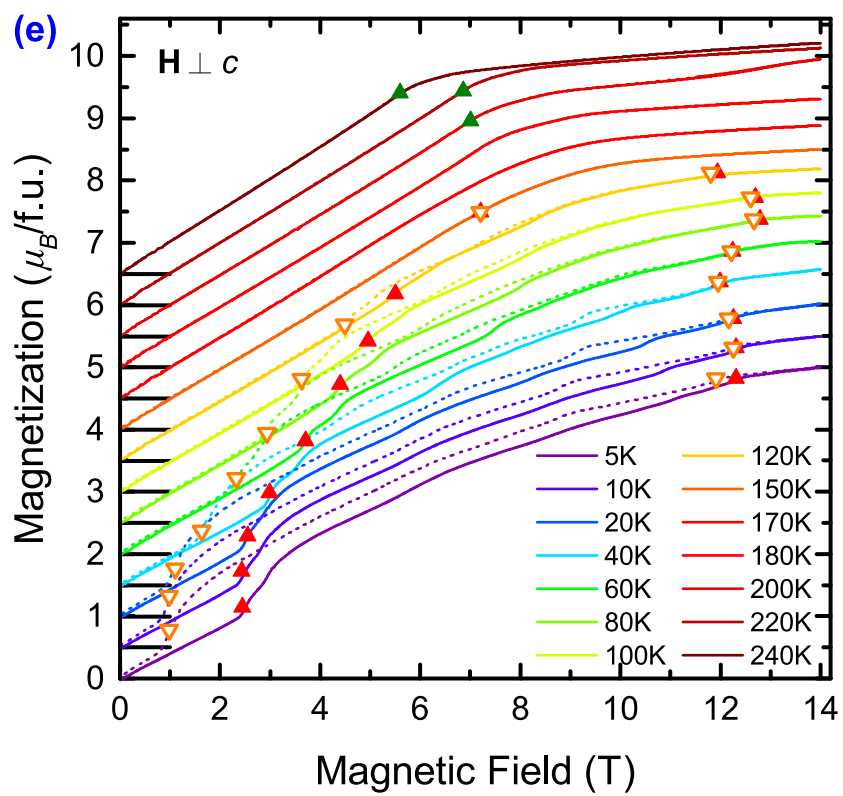

FIG. 6. Magnetic-field dependence of polarization change $\Delta P$ at different temperatures, for (a) $\Delta \mathbf{P} \| c$ and $\mathbf{H} \| c$ axis, (b) $\Delta \mathbf{P} \| c$ and $\mathbf{H} \perp c$ axis, and (c) $\Delta \mathbf{P} \| \mathbf{H}$ and $\mathbf{H} \perp c$ axis. In panels (a), (b), and (c), each polarization curve is shifted vertically by $200 \mu \mathrm{C} / \mathrm{m}^{2}, 200 \mu \mathrm{C} / \mathrm{m}^{2}$, and $100 \mu \mathrm{C} / \mathrm{m}^{2}$, respectively, for the purpose of clarity. $\Delta P$ values marked by cross symbols are plotted in Fig. 7(b) against temperature. (d) Field dependence of the magnetization at various temperatures with magnetic fields applied along the $c$ axis. The inset shows a magnified view of the hysteresis region. (e) Field-dependent magnetization curves at different temperatures when the field is applied perpendicular to $c$. Each curve is shifted vertically by $0.5 \mu_{B} /$ f.u. for better visibility. Data collected in increasing and decreasing magnetic fields are plotted with solid and dashed lines, respectively. The magnetic phase transitions $\mu_{o} H_{\mathrm{C} 1}, \mu_{o} H_{\mathrm{C} 3}$, and $\mu_{o} H_{\mathrm{C} 4}$ are indicated by solid up and empty down triangles for the field increasing and decreasing runs, respectively. These phase transitions are determined as local maximum of the $\frac{d M}{d H}$ curves, and are plotted in the $\mu_{o} H-T$ phase diagram by symbols of the same colors (Fig. 8). For more details about the determination of the phase boundaries see the Supplemental Material [52]. 
perpendicular to the field is negligible. In low fields, the former is proportional to the field and the corresponding linear magnetoelectric coefficient is $\alpha_{c c} \approx 39 \mathrm{ps} / \mathrm{m}$. The hysteresis of the magnetization around zero field is accompanied by a butterfly-shape hysteresis [49] of the polarization [see the inset of Fig. 5(a)]. In the magnetization curve a weak hysteresis is found to persist up to higher fields, as shown in the inset of Fig. 5(c), which is more clearly manifested in the polarization.

Figure 5(b) displays $\Delta P$ when the magnetic field is applied within the $a b$ plane. In this configuration, finite polarizations can be observed in all three independent configurations, though the dominant component is again the one parallel to the easy axis. In this component, the largest changes, as high as $\Delta P \approx-400 \mu \mathrm{C} / \mathrm{m}^{2}$, are observed in the field range of the metamagnetic region. The successive magnetic transitions are accompanied with sudden changes in all three components of the polarization [see the inset of Fig. 5(b)].

Figure 6 shows the three largest terms in the magnetically induced polarization at various temperatures below $T=$ $120 \mathrm{~K}$ together with the corresponding magnetization curves measured for fields along and perpendicular to the easy axis. Figure 6(a) shows the polarization component along the $c$ axis when the magnetic field is applied along the same direction. At each temperature, the polarization shows a butterfly-shape hysteresis at low fields as previously shown in Fig. 5(a) for $T=80 \mathrm{~K}$. The linear magnetoelectric susceptibility at $\mu_{o} H=0 \mathrm{~T}$ shows a sign change at around $T=30 \mathrm{~K}$. The hysteresis is the largest at $T=80 \mathrm{~K}$, and almost vanishes at $T=120 \mathrm{~K}$ similarly to the hysteresis observed in the corresponding magnetization curves [see the inset of Fig. 6(d)].

Figure 6(b) displays the polarization component along the $c$ axis at various temperatures for transverse magnetic field. While the low-field state exhibits negligible polarization change, abrupt variations in the induced polarization occur at the metamagnetic transitions. The hysteresis loops observed in the polarization coincide with those found in the magnetization. Finally, Fig. 6(c) shows the polarization component measured parallel to the magnetic field when both were perpendicular to the $c$ axis. This polarization term is smaller than the other two and exhibits a rather complex field dependence over the range of the metamagnetic transitions.

In Fig. 7(a) we show the temperature evolution of the linear magnetoelectric coefficients $\alpha_{c c}=\partial P_{c} / \partial H_{c}$ and $\alpha_{a a}=$ $\partial P_{a} / \partial H_{a}$. While $\alpha_{c c}$ reaches its maximum value of $\alpha_{c c} \approx$ $39 \mathrm{ps} / \mathrm{m}$ at $T=80 \mathrm{~K}, \alpha_{a a}$ peaks at low temperatures with $\alpha_{a a} \approx 31 \mathrm{ps} / \mathrm{m}$ at $T=20 \mathrm{~K}$. These values are as large as that of $\mathrm{TbPO}_{4}$ with $\alpha_{a a} \approx 37 \mathrm{ps} / \mathrm{m}[50]$ and that of $\mathrm{LiCoPO}_{4}$ with $\alpha_{b a}=30.6 \mathrm{ps} / \mathrm{m} \mathrm{[51].}$

Figure 7(b) displays the temperature dependence of the magnetically induced polarization in constant magnetic fields. Representative field values were chosen to trace thermal features associated with the largest magnetically induced polarization terms. The $c$-axis components of the polarization have maxima at around $T=80 \mathrm{~K}$ irrespective of the orientation of the magnetic field. The polarization component, which is perpendicular to the $c$ axis and parallel to the magnetic field, monotonically decreases with increasing temperature and changes sign at about $80 \mathrm{~K}$.

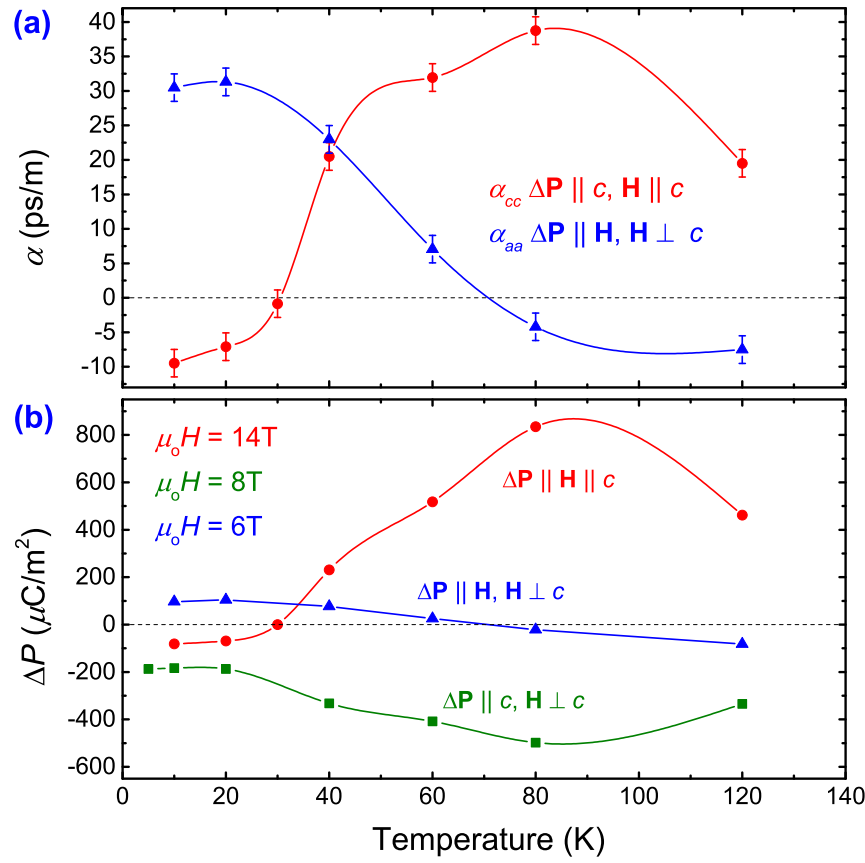

FIG. 7. (a) Temperature dependence of the linear magnetoelectric coefficients $\alpha_{c c}=\frac{d P_{c}}{d H_{c}}$ and $\alpha_{a a}=\frac{d P_{a}}{d H_{a}}$ measured in the $\Delta \mathbf{P} \| \mathbf{H}$ configurations. (b) Temperature evolution of the polarization changes in representative magnetic fields in three measurement configurations. The corresponding values are indicated by crosses in Figs. 6(a)-6(c).

\section{C. $\mu_{o} H-T$ phase diagram}

Based on the results of temperature- and field-dependent magnetization, magnetoelectric, and specific-heat measurements, we have constructed the magnetic phase diagram of $\mathrm{CaBaFe}_{4} \mathrm{O}_{7}$. Figures 8(a) and 8(b) present the $\mu_{0} H-T$ phase diagram for magnetic fields parallel and perpendicular to the $c$ axis, respectively. Below $T_{\mathrm{C} 1}=275 \mathrm{~K}$, we find a ferrimagnetic phase characterized by an easy-axis anisotropy. In finite magnetic fields applied parallel to the $c$ axis, the paramagnetic to ferrimagnetic transition becomes a crossover, which shifts to higher temperatures with increasing magnetic field. Color gradation is used to indicate the finite-field crossover region between the high-temperature paramagnetic (PM) state and the partially field polarized but still paramagnetic state at lower temperatures. In zero magnetic field this crossover becomes a well defined transition between the PM state and the FiM1 ferrimagnetic state at $T_{\mathrm{C} 1}=275 \mathrm{~K}$. In contrast, for magnetic fields perpendicular to the $c$ axis the FiM1 easy-axis ferrimagnet phase extends to finite fields and it is separated from the field-polarized PM phase by the $\mu_{o} H_{\mathrm{Cl}}(T)$ phase boundary, which is rapidly suppressed in finite fields.

The spin reorientation transition from the FiM1 phase to another ferrimagnetic phase (FiM2) at around $T_{\mathrm{C} 2}=211 \mathrm{~K}$ is resolved in the magnetization as well as in the specific-heat measurements. In finite magnetic fields the FiM2 phase is separated from the FPM and the FiM1 phases by the transition lines labeled as $\mu_{o} H_{\mathrm{C} 2}^{\|}(T)$ and $\mu_{o} H_{\mathrm{C} 2}^{\perp}(T)$ for fields parallel and perpendicular to the $c$ axis, respectively. Here we note that $\mu_{o} H_{\mathrm{C} 2}^{\perp}(T)$ shows up only in specific-heat data and remains indiscernible in the magnetization measurements in low fields, 

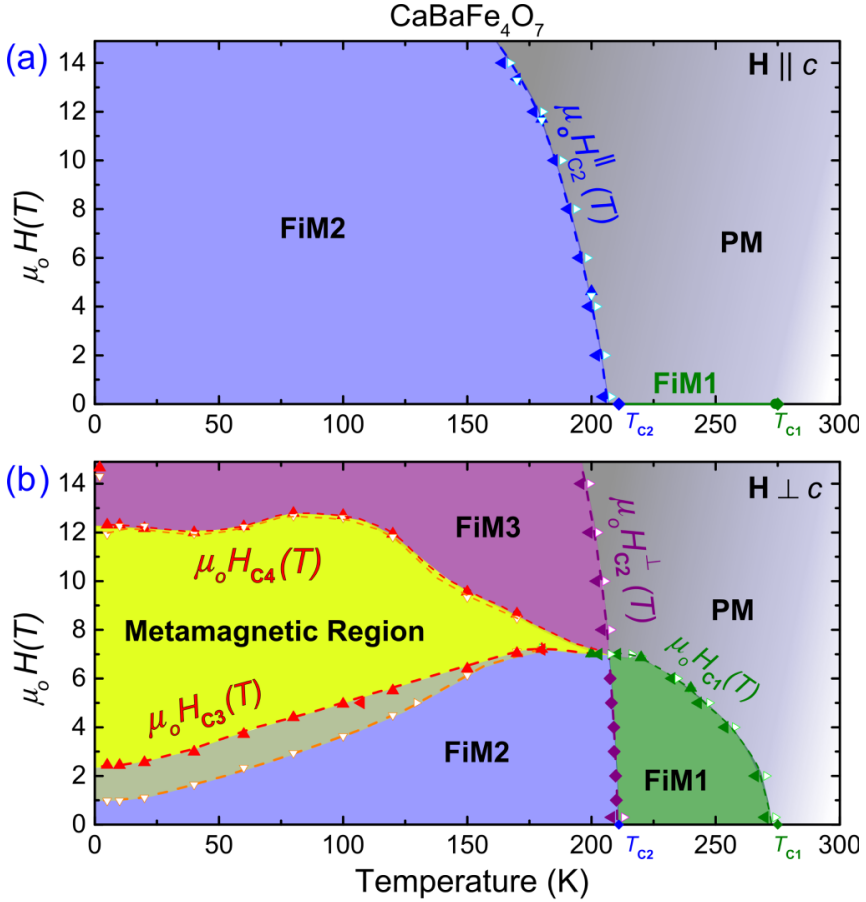

FIG. 8. Temperature versus magnetic field phase diagram for fields applied (a) parallel and (b) perpendicular to the $c$ axis. Phase boundaries based on magnetization measurements are represented by full or empty triangles, while those obtained from specific-heat measurements are denoted by diamonds. Direction of the triangles illustrates the following measurement conditions: triangles pointing up and down correspond to isothermal magnetization measurements with increasing and decreasing fields, respectively, while triangles pointing left and right indicate magnetization measurements at fixed external fields measured with decreasing and increasing temperatures, respectively. The phase boundaries are represented by dashed lines as a guide to the eyes. The finite-field crossover region where the spin polarization rapidly develops in the paramagnetic state is indicated by color gradation. For a detailed discussion of the different phases and the determination of the phase boundaries see the main text and the Supplemental Material [52].

namely up to its crossing with $\mu_{o} H_{\mathrm{C} 1}(T)$. As discussed earlier and documented in Fig. 6 in detail, for $T<T_{\mathrm{C} 2}$ there is a sequence of metamagnetic transitions located below $\mu_{o} H_{\mathrm{C} 2}^{\perp}(T)$, when the magnetic field is applied perpendicular to the $c$ axis. This metamagnetic region is bound by the $\mu_{o} H_{\mathrm{C} 3}(T)$ and $\mu_{o} H_{\mathrm{C} 4}(T)$ lines and separates the low-field FiM2 and the high-field FiM3 phases, the latter of which is still ferrimagnetic with a magnetization about three times lower than what would correspond to the fully polarized ferromagnetic state. The transition line $\mu_{o} H_{\mathrm{C} 3}(T)$ is characterized by a wide hysteresis.

\section{CONCLUSIONS}

Melt-grown crystals of the orthorhombic polar ferrimagnet $\mathrm{CaBaFe}_{4} \mathrm{O}_{7}$ have been synthesized with the optical floatingzone technique. Based on the combined magnetization, calorimetric, and magnetoelectric measurements, we have mapped out the $\mu_{o} H-T$ magnetic phase diagram of the material. Specific-heat measurements revealed that a structural phase transition to a higher symmetry form of $\mathrm{CaBaFe}_{4} \mathrm{O}_{7}$ takes place at $T_{\mathrm{S}}=380 \mathrm{~K}$. We found a paramagnetic to ferrimagnetic transition at $T_{\mathrm{C} 1}=275 \mathrm{~K}$ followed by a spin reordering transition at $T_{\mathrm{C} 2}=211 \mathrm{~K}$. Below $T_{\mathrm{C} 2}$ magnetic fields applied perpendicular to the $c$ axis drive the system through a cascade of metamagnetic transitions. While the spin patterns in the different phases cannot be determined from the present data, we explored the magnetic phase diagram in fine detail for magnetic fields applied both along and perpendicular to the easy axis. In addition, we have investigated the magnetoelectric properties below $T_{\mathrm{C} 2}$ with different orientations of the magnetic field. A large linear magnetoelectric coefficient of $\alpha_{c c} \approx 39 \mathrm{ps} / \mathrm{m}$ as well as a gigantic field-induced polarization of $\Delta P=850 \mu \mathrm{C} / \mathrm{m}^{2}$ have been observed for magnetic fields along the $c$ axis. The magnetic phase diagram together with the observed magnetoelectric effect establishes a good starting point to develop a microscopic description of the coupling between spin and charge degrees of freedom in $\mathrm{CaBaFe}_{4} \mathrm{O}_{7}$, a near-room-temperature multiferroic oxide. However, the low symmetry of the crystal structure does not allow the identification of the microscopic origin of the magnetoelectric coupling at the present stage. Determination of the magnetic structure in the different magnetic phases together with ab initio calculations could complement our study to assign the microscopic origin of multiferroicity [7], namely whether the magnetically induced polarization is governed by magnetostriction, by the spin-current mechanism, or by spin-dependent orbital hybridization between the irons and their oxygen ligands.

\section{ACKNOWLEDGMENTS}

The authors are grateful for the technical assistance provided by A. Kikkawa and T. Suzuki, and for the fruitful discussion with I. Lovas. This work was supported by the Funding Program for World-Leading Innovative R\&D on Science and Technology (FIRST Program) on "Quantum Science on Strong Correlation" from JSPS and by the Hungarian Research Funds OTKA K 108918, OTKA PD 111756, and Bolyai 00565/14/11. We acknowledge the support of the HFML-RU/FOM, member of the European Magnetic Field Laboratory (EMFL). M.K. is supported by a Grant-in-Aid for Young Scientists (B) (JSPS, KAKENHI No. 25800197) and by a Grant-in-Aid for Scientific Research (C) (JSPS, KAKENHI No. 15K05140).
[1] D. N. Astrov, Sov. Phys. JETP 13, 729 (1961).

[2] T. Kimura, T. Goto, H. Shintani, K. Ishizaka, T. Arima, and Y. Y. Tokura, Nature (London) 426, 55 (2003).

[3] N. Hur, P. A. Sharma, J. S. Ahn, S. Guha, and S.-W. Cheong, Nature (London) 429, 392 (2004).
[4] M. Fiebig, J. Phys. D: Appl. Phys. 38, R123 (2005).

[5] S.-W. Cheong and M. Mostovoy, Nat. Mater. 6, 13 (2007).

[6] Y. Tokunaga, Y. Taguchi, T.-h. Arima, and Y. Tokura, Nat. Phys. 8, 838 (2012). 
[7] Y. Tokura, S. Seki, and N. Nagaosa, Rep. Prog. Phys. 77, 076501 (2014).

[8] R. Ramesh and N. A. Spaldin, Nat. Mater. 6, 21 (2007).

[9] L. W. Martin, Y.-H. Chu, and R. Ramesh, Mater. Sci. Eng., R 68, 89 (2010).

[10] I. Kézsmárki, D. Szaller, S. Bordács, V. Kocsis, Y. Tokunaga, Y. Taguchi, H. Murakawa, Y. Tokura, H. Engelkamp, T. Rõõm, and U. Nagel, Nat. Commun. 5, 3203 (2014).

[11] I. Kézsmárki, U. Nagel, S. Bordács, R. S. Fishman, J. H. Lee, H. T. Yi, S.-W. Cheong, and T. Rõõm, Phys. Rev. Lett. 115, 127203 (2015).

[12] V. E. Wood and A. E. Austin, Int. J. Magn. 5, 303 (1973).

[13] H. Murakawa, Y. Onose, S. Miyahara, N. Furukawa, and Y. Tokura, Phys. Rev. Lett. 105, 137202 (2010).

[14] M. Akaki, H. Iwamoto, T. Kihara, M. Tokunaga, and H. Kuwahara, Phys. Rev. B 86, 060413 (2012).

[15] S. Bordács, I. Kézsmárki, D. Szaller, L. Demkó, N. Kida, H. Murakawa, Y. Onose, R. Shimano, T. Rõõm, U. Nagel, S. Miyahara, N. Furukawa, and Y. Tokura, Nat. Phys. 8, 734 (2012).

[16] T. Kimura, Annu. Rev. Condens. Matter Phys. 3, 93 (2012).

[17] I. Kézsmárki, S. Bordács, P. Milde, E. Neuber, L. M. Eng, J. S. White, H. M. Rønnow, C. D. Dewhurst, M. Mochizuki, K. Yanai, H. Nakamura, D. Ehlers, V. Tsurkan, and A. Loidl, Nat. Mater. 14, 1116 (2015).

[18] E. Ruff, S. Widmann, P. Lunkenheimer, V. Tsurkan, S. Bordács, I. Kézsmárki, and A. Loidl, Sci. Adv. 1, e1500916 (2015).

[19] J. Wang, J. B. Neaton, H. Zheng, V. Nagarajan, S. B. Ogale, B. Liu, D. Viehland, V. Vaithyanathan, D. G. Schlom, U. V. Waghmare, N. A. Spaldin, K. M. Rabe, M. Wuttig, and R. Ramesh, Science 299, 1719 (2003).

[20] S. Lee, W. Ratcliff, S.-W. Cheong, and V. Kiryukhin, Appl. Phys. Lett. 92, 192906 (2008).

[21] M. Tokunaga, M. Akaki, T. Ito, S. Miyahara, A. Miyake, H. Kuwahara, and N. Furukawa, Nat. Commun. 6, 5878 (2015).

[22] V. Caignaert, V. Pralong, A. Maignan, and B. Raveau, Solid State Commun. 149, 453 (2009).

[23] N. Hollmann, Z. Hu, H. Wu, M. Valldor, N. Qureshi, T. Willers, Y.-Y. Chin, J. C. Cezar, A. Tanaka, N. B. Brookes, and L. H. Tjeng, Phys. Rev. B 83, 180405 (2011).

[24] K. Singh, V. Caignaert, L. C. Chapon, V. Pralong, B. Raveau, and A. Maignan, Phys. Rev. B 86, 024410 (2012).

[25] V. Caignaert, A. Maignan, K. Singh, C. Simon, V. Pralong, B. Raveau, J. F. Mitchell, H. Zheng, A. Huq, and L. C. Chapon, Phys. Rev. B 88, 174403 (2013).

[26] R. D. Johnson, K. Cao, F. Giustino, and P. G. Radaelli, Phys. Rev. B 90, 045129 (2014).

[27] V. Caignaert, A. M. Abakumov, D. Pelloquin, V. Pralong, A. Maignan, G. V. Tendeloo, and B. Raveau, Chem. Mater. 21, 1116 (2009).
[28] W. Schweika, M. Valldor, and P. Lemmens, Phys. Rev. Lett. 98, 067201 (2007).

[29] H. Hao, C. Chen, L. Pan, J. Gao, and X. Hu, Physica B (Amsterdam, Neth.) 387, 98 (2007).

[30] N. Nakayama, T. Mizota, Y. Ueda, A. Sokolov, and A. Vasiliev, J. Magn. Magn. Mater. 300, 98 (2006).

[31] H. Hao, Q. He, Y. Cheng, and L. Zhao, J. Phys. Chem. Solids 75, 495 (2014).

[32] V. Caignaert, V. Pralong, V. Hardy, C. Ritter, and B. Raveau, Phys. Rev. B 81, 094417 (2010).

[33] S. Chatterjee and T. Saha-Dasgupta, Phys. Rev. B 84, 085116 (2011).

[34] A. Huq, J. Mitchell, H. Zheng, L. Chapon, P. Radaelli, K. Knight, and P. Stephens, J. Solid State Chem. 179, 1136 (2006).

[35] A. Maignan, V. Caignaert, D. Pelloquin, S. Hébert, V. Pralong, J. Hejtmanek, and D. Khomskii, Phys. Rev. B 74, 165110 (2006).

[36] V. Caignaert, A. Maignan, V. Pralong, S. Hébert, and D. Pelloquin, Solid State Sci. 8, 1160 (2006).

[37] D. D. Khalyavin, P. Manuel, B. Ouladdiaf, A. Huq, P. W. Stephens, H. Zheng, J. F. Mitchell, and L. C. Chapon, Phys. Rev. B 83, 094412 (2011).

[38] M. Valldor, Solid State Sciences 6, 251 (2004).

[39] M. Valldor, N. Hollmann, J. Hemberger, and J. A. Mydosh, Phys. Rev. B 78, 024408 (2008).

[40] M. Soda, Y. Yasui, T. Moyoshi, M. Sato, N. Igawa, and K. Kakurai, J. Phys. Soc. Jpn. 75, 054707 (2006).

[41] L. C. Chapon, P. G. Radaelli, H. Zheng, and J. F. Mitchell, Phys. Rev. B 74, 172401 (2006).

[42] T. Sarkar, V. Pralong, V. Caignaert, and B. Raveau, Chem. Mater. 22, 2885 (2010).

[43] K. Momma and F. Izumi, J. Appl. Cryst. 41, 653 (2008).

[44] M. Valldor, R. P. Hermann, J. Wuttke, M. Zamponi, and W. Schweika, Phys. Rev. B 84, 224426 (2011).

[45] M. Valldor and O. Breunig, Solid State Sci. 13, 831 (2011).

[46] T. Sarkar, V. Caignaert, V. Pralong, and B. Raveau, J. Supercond. Novel Magn. 25, 627 (2012).

[47] B. Raveau, V. Caignaert, V. Pralong, D. Pelloquin, and A. Maignan, Chem. Mater. 20, 6295 (2008).

[48] K. Vijayanandhini, C. Simon, V. Pralong, V. Caignaert, and B. Raveau, Phys. Rev. B 79, 224407 (2009).

[49] E. Ascher, H. Rieder, H. Schmid, and H. Stössel, J. Appl. Phys. 37, 1404 (1966).

[50] G. T. Rado, J. M. Ferrari, and W. G. Maisch, Phys. Rev. B 29, 4041 (1984).

[51] I. Kornev, M. Bichurin, J.-P. Rivera, S. Gentil, H. Schmid, A. G. M. Jansen, and P. Wyder, Phys. Rev. B 62, 12247 (2000).

[52] See Supplemental Material at http://link.aps.org/supplemental/ 10.1103/PhysRevB.93.014444 for a detailed description of the magnetic phases and the determination of the phase boundaries. 